\title{
Bottomonium melting from screening correlators at high temperature
}

\author{
Peter Petreczky $\odot$ \\ Physics Department, Brookhaven National Laboratory, Upton, New York 11973, USA \\ Sayantan Sharma \\ The Institute of Mathematical Sciences, HBNI, Chennai 600113, India \\ Johannes Heinrich Weber® \\ Institut für Physik, Humboldt-Universität zu Berlin \& IRIS Adlershof, D-12489 Berlin, Germany
}

(Received 7 August 2021; accepted 6 September 2021; published 24 September 2021)

\begin{abstract}
We study the bottomonium screening masses in a $2+1$ flavor QCD on the lattice using the highly improved staggered quark (HISQ) action. We focus on a wide temperature range in the region $350 \mathrm{MeV} \leq T<1000 \mathrm{MeV}$, and perform our calculations on three different lattice spacings corresponding to temporal lattice extent of $N_{\tau}=8,10$ and 12, in order to control the lattice cutoff effects. From a detailed study of the temperature dependence of screening masses we conclude that the $\eta_{b}(1 S)$ and $\Upsilon(1 S)$ states melt at $T>500 \mathrm{MeV}$, while the scalar and axial-vector states $\chi_{b 0}(1 P)$ and $h_{b}(1 P)$ melt already at $T>350 \mathrm{MeV}$.
\end{abstract}

DOI: 10.1103/PhysRevD.104.054511

\section{INTRODUCTION}

The study of the properties of bound states of heavy quarks called quarkonium has received a lot of attention since the seminal paper by Matusi and Satz [1], where it was suggested that the formation of a color deconfined medium in heavy-ion collisions will lead to the dissolution of quarkonium, resulting in a suppression of their production. There are large experimental efforts in recent times dedicated towards understanding quarkonium production and its dynamics in heavy-ion collisions, which are supplemented by many phenomenological studies, see Refs. [2-7] for recent reviews.

The in-medium properties of quarkonium in a quarkgluon plasma (QGP) as well as its dissolution as a function of the temperature are all encoded in the quarkonium spectral functions, that are defined in terms of the real-time correlation functions of the appropriate hadron operators. Quarkonium states show up as peaks in the spectral functions, which at high temperatures are expected to be broadened and shifted in the frequency space, and eventually merge into the continuum of quark-antiquark scattering states. Through an analytic continuation one can relate the spectral function of the quarkonium state of a specific

Published by the American Physical Society under the terms of the Creative Commons Attribution 4.0 International license. Further distribution of this work must maintain attribution to the author(s) and the published article's title, journal citation, and DOI. Funded by SCOAP ${ }^{3}$. quantum number channel to the Euclidean time correlation function, which can be calculated using lattice field theory techniques. The Euclidean time correlation function is the Laplace transform of the spectral function, if thermal particle production can be neglected, and has a more complicated kernel otherwise. Therefore, lattice calculations can in principle provide a model independent information on the quarkonium spectral functions. However, in practice the reconstruction of the spectral function from a discrete set of Euclidean correlator data from lattice is a difficult task. Early works on the reconstruction of the spectral functions have been reported in Refs. [8-15]. It has also been pointed out that the Euclidean correlation functions have limited sensitivity to the in-medium quarkonium properties and/or their melting [16,17], because at high temperatures the temporal extent of the lattice becomes small. In the case of bound states of bottom quarks (bottomonium) there is an additional problem of large discretization errors in the correlators due to the large bottom-quark mass. One can circumvent this problem by using nonrelativistic QCD (NRQCD), an effective theory in which the energy scale associated with the heavy quark mass has been integrated out [18]. This approach is widely used to calculate bottomonium properties at zero temperature [19-24]. Recent studies within the NRQCD formalism at finite temperature [25-30] have indicated that the ground states of bottomonium channels, $\Upsilon(1 S)$ and $\eta_{b}(1 S)$, can survive up to temperatures of $400 \mathrm{MeV}$, whereas the fate of $P$-wave bottomonia is not yet completely settled. Lattice results from the FASTUM collaboration suggest 
that the $P$-wave states melt already at temperatures around $200 \mathrm{MeV}$ [26], while other independent studies also within the NRQCD formalism suggest that $P$-wave bottomonia can survive at higher temperatures within the QGP [28-30]. Since NRQCD is an effective theory with an ultraviolet cutoff of around the bottom quark mass, the choice of lattice spacings in these calculations cannot be too small. Since the temperature is related to the inverse lattice spacing, $T=1 /\left(a N_{\tau}\right)$ with $N_{\tau}$ being the temporal extent, this also suggests that studying bottomonia at high temperature with some reasonable choice of $N_{\tau}$ is difficult. For this reason we do not know yet at what temperatures the ground state bottomonia melt.

The spatial correlation functions of mesons can offer a different perspective on the problem of in-medium modification of mesons, in particular charmonium [31-33]. In contrast to the temporal meson correlators, the spatial meson correlation functions can be calculated for large (spatial) separations between the source and the sink and, therefore, are more sensitive to the in-medium modifications of meson states [31-33]. The spatial correlation functions are in turn related to the meson spectral function at nonzero momenta through the relation

$$
G(z, T)=\int_{0}^{\infty} \frac{2 d \omega}{\omega} \int_{-\infty}^{\infty} d p_{z} e^{i p_{z} z} \sigma\left(\omega, p_{z}, T\right) .
$$

While the above relation is more complicated than the corresponding relation for the temporal correlator and spectral function for mesons, it is still quite useful. At large distances the spatial meson correlation function decays exponentially, and the exponential decay is governed by the so-called screening mass, $G(z) \sim \exp \left(-M_{\text {scr }} \mathrm{z}\right)$. When there is a well-defined bound state peak in the meson spectral function, the screening mass will be equal to the meson pole mass $[31,32]$. On the other hand at very high temperatures, when the quark and antiquarks are eventually unbound, the screening mass is given by $2 \sqrt{(\pi T)^{2}+m_{q}^{2}}$, with $m_{q}$ being the quark mass. Thus the temperature dependence of the meson screening masses can provide some valuable information about the melting of meson states. The analysis of the spatial correlation functions have provided some evidence for sequential in-medium modification of different charmonium states, i.e., stronger in-medium modification of excited charmonia compared to its ground states, and for the dissolution of the $1 S$ charmonium state at temperatures $T>300 \mathrm{MeV}$ [32].

The aim of this paper is to provide some new insights on the melting of bottomonium states in the QGP through the study of their spatial correlation functions. For the first time we use the full relativistic Dirac operator for the bottom quarks in the construction of the meson correlators in $2+1$ flavor QCD, which allows us to make an independent prediction on the melting of different quantum number states independent of the NRQCD formalism. We can thus unambiguously observe an earlier melting of the scalar and axial-vector bottomonium states compared to the pseudoscalar and vector channels. The paper is organized as follows. In Sec. II we provide the details of the techniques we use. Subsequently the main results on the bottomonium screening masses are discussed in Sec. III, followed by our concluding section.

\section{LATTICE SETUP}

We calculate the screening masses of the bottomonium states in QCD with $2+1$ flavors of dynamical quarks treating the bottom quarks in the quenched approximation. We use the highly improved staggered quark (HISQ) action [34] for the quarks and a tree level Symanzik improved gauge action. Using HISQ action for the valence bottom quark is important since it preserves the correct dispersion relation for heavy quarks [34]. The strange quark mass, $m_{s}$, was chosen to be close to its physical value, while the light quark masses $m_{l}=m_{s} / 20$ correspond to a pion mass of $160 \mathrm{MeV}$ in the continuum limit [35]. We perform our calculations on $N_{\sigma}^{3} \times N_{\tau}$ lattice with temporal extent of $N_{\tau}=8,10,12$ and the spatial extent, $N_{\sigma}$ fixed by $N_{\sigma}=4 N_{\tau}$. The corresponding gauge configurations have been generated by the HotQCD collaboration [35-37]. We have specifically focused on a wide temperature range between 2-8 $T_{c}$, where $T_{c}=156.5(1.5) \mathrm{MeV}$ is the chiral crossover temperature [38]. This enables us to measure the full details of the thermal evolution of the bottomonium correlators. Moreover we ensured that $m_{b} a \lesssim 1$ for the lattice spacings over this entire temperature range of interest, which in turn allowed us to have sufficient control on the lattice artifacts in the results of the bottomonium correlators. Having three different lattice extents allowed us to have a better control on the discretization effects at high temperatures. The bottom-quark mass in this entire range was set to be $52.5 m_{s}$, which is close to its physical value. The lattice spacing was determined in physical units using the $r_{1}$ scale defined in terms of the static quark-antiquark potential through $\left.r^{2} \frac{d V(r)}{d r}\right|_{r=r_{1}}=1$. We used the parametrization of $a / r_{1}$ obtained in Ref. [39] and the value $r_{1}=$ $0.3106(18) \mathrm{fm}$ [40]. The details of the lattice parameters including the bare lattice gauge coupling $\beta=10 / g_{0}^{2}$, the quark masses, the temperatures as well as the number of configurations used in this work are summarized in Table I.

The meson operators for staggered fermions have the form

$J_{M}(\mathbf{x})=\bar{q}(\mathbf{x})\left(\Gamma_{D} \times \Gamma_{F}\right) q(\mathbf{x}), \quad \mathbf{x}=(x, y, z, \tau)$,

where $\Gamma_{D}, \Gamma_{F}$ are the Dirac gamma matrices corresponding to the spin and the staggered taste (flavor) structure. In this work we consider the case where $\Gamma_{D}=\Gamma_{F}=\Gamma$. This choice corresponds to local operators for the meson currents, which in terms of the staggered quark fields have 
TABLE I. The gauge coupling, $\beta$, the quark masses, the temperature values and the number of gauge configurations (\#c) used in this study.

\begin{tabular}{|c|c|c|c|c|c|c|c|c|}
\hline \multirow[b]{2}{*}{$\beta$} & \multirow[b]{2}{*}{$a m_{s}$} & \multirow[b]{2}{*}{$a m_{b}$} & \multicolumn{2}{|c|}{$N_{\tau}=8$} & \multicolumn{2}{|c|}{$N_{\tau}=10$} & \multicolumn{2}{|c|}{$N_{\tau}=12$} \\
\hline & & & $T$ & $\# \mathrm{c}$ & $T$ & $\# \mathrm{c}$ & $T$ & \#c \\
\hline 7.650 & 0.0192 & 1.0090 & $\cdots$ & $\cdots$ & $\ldots$ & $\cdots$ & 350 & 220 \\
\hline 7.825 & 0.0164 & 0.8618 & 611 & 500 & 489 & 250 & 407 & 180 \\
\hline 8.000 & 0.0140 & 0.7357 & 711 & 500 & 571 & 500 & 476 & 180 \\
\hline 8.200 & 0.0117 & 0.6133 & 844 & 250 & 675 & 250 & 562 & 500 \\
\hline 8.400 & 0.0098 & 0.5124 & 1000 & 240 & 800 & 250 & 666 & 500 \\
\hline 8.570 & 0.0084 & 0.4402 & $\ldots$ & $\ldots$ & 923 & 250 & 769 & 250 \\
\hline 8.710 & 0.0074 & 0.3886 & $\ldots$ & $\ldots$ & $\ldots$ & $\ldots$ & 866 & 250 \\
\hline 8.850 & 0.0065 & 0.3431 & $\ldots$ & $\cdots$ & $\ldots$ & $\ldots$ & 974 & 250 \\
\hline
\end{tabular}

the simple form $J_{M}(\mathbf{x})=\tilde{\phi}(\mathbf{x}) \bar{\chi}(\mathbf{x}) \chi(\mathbf{x})$. The staggered phase $\tilde{\phi}(\mathbf{x})$ specifies the quantum numbers of the meson channel. In this work we consider the spatial correlation functions along the $z$-direction:

$$
C_{M}(z)=\int d x d y d \tau\left\langle J_{M}(\mathbf{x}) J_{M}(0)\right\rangle .
$$

For each choice of $\tilde{\phi}(\mathbf{x})$, the staggered meson correlation function contains contributions from both parity states, which correspond to the oscillating and nonoscillating parts of the correlators. If we restrict ourselves to the lowest states, the spatial meson correlation function can be simply written as

$$
\begin{aligned}
C_{M}(z)= & A_{N O} \cosh \left[M_{N O}\left(z-\frac{N_{s}}{2}\right)\right] \\
& -(-1)^{z} A_{O} \cosh \left[M_{O}\left(z-\frac{N_{s}}{2}\right)\right] .
\end{aligned}
$$

The subscripts $O$ and $N O$ for the screening masses and amplitudes denote the oscillating and nonoscillating states. In Table II we give the details of the staggered phases corresponding to the oscillating and nonoscillating contributions for different meson quantum numbers, as well as the labels denoting the screening masses in the pseudoscalar (PS), scalar (S), vector (V) and the axial-vector (AV) channels. In this study we used point sources

TABLE II. The staggered phases, the $\Gamma$ matrices, the bottomonium states and the corresponding screening masses considered in this study.

\begin{tabular}{lccccc}
\hline \hline & $-\tilde{\phi}(\mathbf{x})$ & $\Gamma$ & $J^{P C}$ & Meson & Screening mass \\
\hline$M_{N O}$ & 1 & $\gamma_{4} \gamma_{5}$ & $0^{-+}$ & $\eta_{b}$ & $M_{\text {scr }}^{P S}$ \\
$M_{O}$ & & 1 & $0^{++}$ & $\chi_{b 0}$ & $M_{\text {scr }}^{S}$ \\
$M_{N O}$ & $(-1)^{x+\tau},(-1)^{y+\tau}$ & $\gamma_{i}$ & $1^{--}$ & $\Upsilon$ & $M_{\text {scr }}^{V}$ \\
$M_{O}$ & $\gamma_{j} \gamma_{k}$ & $1^{+-}$ & $h_{b}$ & $M_{\text {scr }}^{A V}$ \\
\hline \hline
\end{tabular}

corresponding to the quark and antiquarks in the meson correlators and performed two state fits of the corresponding correlators to Eq. (4) in order to determine the bottomonium screening masses.

\section{RESULTS}

We begin by showing the pseudoscalar $\eta_{b}$ screening mass as a function of the temperature, in Fig. 1. The results on the screening mass at each temperature have been normalized by the zero temperature $\eta_{b}$ meson mass in this figure. While the ratio $m_{b} / m_{s}$ is chosen close to its physical value, the lines of constant physics for the strange quark mass have not been fixed very precisely for this temperature range [35]. Therefore, we cannot use the experimentally measured mass for $\eta_{b}$ from the Particle Data Group [41], and need to take into account the small deviations of the $b$ quark mass from its physical value. The dependence of the $\eta_{b}$ meson mass on the $b$ quark mass for the HISQ action has been studied earlier in Ref. [42] for $\beta=7.596$, $7.825,8.0,8.2$ and 8.4. Therefore, we could estimate the zero temperature $\eta_{b}$ mass for $\beta=7.825,8.0,8.2$ and 8.4 and the $b$ quark masses given in Table I. It turns out that the $\eta_{b}$ mass is larger than the PDG value by $9.8 \%$ for $\beta=8.4$. For $\beta>8.4$, where we do not have the zero temperature mass data, we assume that the $\eta_{b}$ mass is $9.8 \%$ larger than the experimentally measured value based on the above result. By fitting the lattice $b$ quark mass that corresponds to the physical value for $\beta=7.596,7.825,8.0,8.2$ and 8.4 [42] with the renormalization group inspired Ansatz we determine that the input $b$ quark mass for $\beta=7.65$ is $5.5 \%$ larger than its physical value. We can also estimate that at the close-by value of $\beta$, namely $\beta=7.596$, the $5.5 \%$ larger $b$-quark mass leads to an $\eta_{b}$ meson mass that is $4.4 \%$ larger than the physical value. Therefore, we assume that the $\eta_{b}$ meson mass for $\beta=7.65$ is $4.4 \%$ larger than the PDG

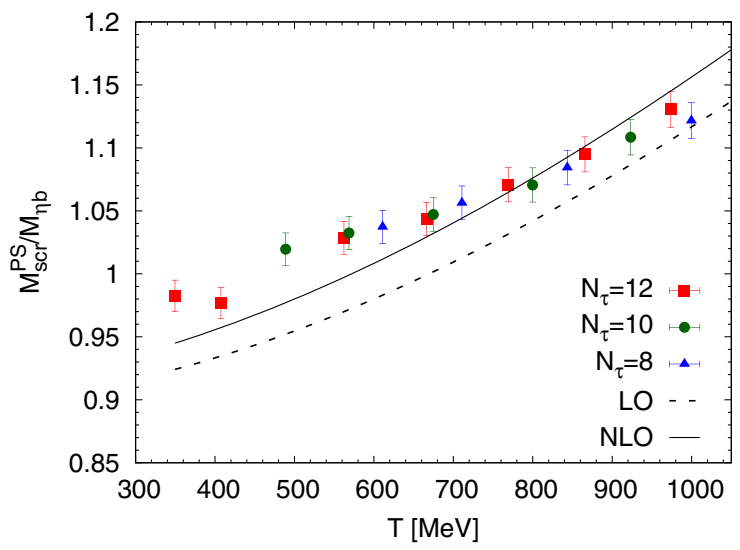

FIG. 1. The pseudoscalar screening mass divided by the mass of the $\eta_{b}(1 S)$ meson at zero temperature as a function of the temperature obtained on lattices with $N_{\tau}=8,10$ and 12 . The solid line is LO prediction for the screening mass, while the dashed line is the NLO prediction, see text. 
value. Since we did not calculate the zero temperature $\eta_{b}$ masses explicitly on the given lattice spacings but estimated them based on the interpolation, we assign a systematic error of $1 \%$ to all zero temperature $\eta_{b}$ masses to account for possible systematic effects. For the data in Fig. 1, we also consider the errors in the scale setting $a / r_{1}$ as well as the error of $r_{1}$ in physical units. Different sources of errors have been added in quadrature to determine the error on each data point. We observe that the lattice cutoff $\left(N_{\tau}\right.$ dependence) of the results shown in Fig. 1 is small compared to the estimated systematic and statistical errors.

In Fig. 1 we observe that at the lowest temperatures, the $\eta_{b}$ screening mass is close to the zero temperature mass, while at high temperature the screening mass increases roughly linearly with the temperature. The temperature dependence of the $\eta_{b}$ screening mass is qualitatively very similar to the temperature dependence of the ground state charmonium $\left(\eta_{c}\right.$ and $\left.J / \psi\right)$ screening masses, except that for charmonium the linear increase with the temperature is seen already at $T>250 \mathrm{MeV}$ [32]. We recall here that the approximately linear increase of the screening masses with temperature corresponds to an unbound quark-antiquark pair, where the screening mass at leading order (LO) for quarks of mass $m_{q}$ is given by $2 \sqrt{(\pi T)^{2}+m_{q}^{2}}$. The nextto-leading correction to the screening mass has also been calculated [43]. Therefore, we will also use the next-toleading (NLO) result to compare with the lattice data. We show both the LO and NLO result for the bottomonium screening mass in Fig. 1. For the bottom quarks we use the $\overline{\mathrm{MS}}$ mass at the scale $\mu=m_{b}$, given by $m\left(\mu=m_{b}\right)=$ $4.188 \mathrm{GeV}$ [42]. We observe that the lattice results for the pseudoscalar screening mass are close to the NLO predictions for $T>500 \mathrm{MeV}$. The temperature dependence of the pseudoscalar screening masses for $T>500 \mathrm{MeV}$ suggests that the bottom quark and antiquark are no longer consistently bound, i.e., the $\eta_{b}$ meson melting is under way at $T>500 \mathrm{MeV}$. At lower temperatures the $\eta_{b}$ state exists with small in-medium modifications. The latter conclusion is consistent with the findings from NRQCD based studies $[25,26,28-30]$ as well as with the results obtained from potential models with a screened complex potential $[44,45]$.

Next we study the temperature dependence of the difference between the vector $\Upsilon$ and $\eta_{b}$ screening masses, which is shown in Fig. 2. We do not expect this difference to be affected by the small deviations of the $b$-quark mass from its physical value, and therefore, we do not attempt to correct for these small deviations. In estimating the errors for this observable, we have simply added the errors in the determination of the lattice spacings and the statistical errors in quadrature. We again observe a mild $N_{\tau}$ dependence of the results compared to the estimated errors. At zero temperature the difference between the $\Upsilon(1 S)$ and $\eta_{b}(1 S)$ mass is about $70 \mathrm{MeV}$ [41], and is caused by spin-

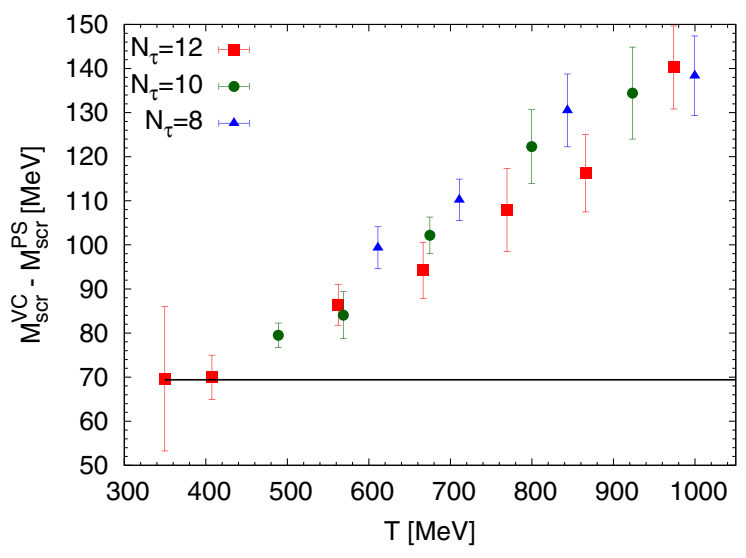

FIG. 2. The difference between the vector and pseudoscalar screening masses as a function of the temperature obtained on lattices with $N_{\tau}=8,10$ and 12 . The solid line corresponds to the difference between the $\Upsilon(1 S)$ mass and the $\eta_{b}(1 S)$ mass from the Particle Data Group (PDG) [41].

dependent interactions, which are suppressed as $1 / m_{b}^{2}$. At the lowest two temperatures the difference between the vector and pseudoscalar screening masses is consistent with this value. This suggests that at these temperatures the $\eta_{b}(1 S)$ and $\Upsilon(1 S)$ exist as well-defined bound states with possibly little in-medium modifications. For $T \geq 500 \mathrm{MeV}$ this difference increases linearly with temperature. Perturbative calculation at NLO in the strong-coupling constant predicts this difference to be identically zero. In order to understand the linear temperature dependence of the difference between the vector and pseudoscalar screening masses at high temperatures one has to go beyond NLO, and instead consider a dimensionally reduced three-dimensional effective theory of QCD. Within this effective theory, a quark and antiquark propagating along the $z$-direction interact via a spin-dependent potential which is proportional to the temperature $[46,47]$. This spin dependent potential causes a splitting between the pseudoscalar and vector screening mass that is also proportional to the temperature [46,47]. For light quarks, where the effect of their masses is negligible, this feature has been observed in the lattice calculations for $T>900 \mathrm{MeV}$, and the difference is $\sim 0.3 T$ [48]. For bottom quarks, however, the effective quark mass in the effective threedimensional theory is larger, resulting in the suppression of the spin-dependent interactions. As a result, the difference between the vector and pseudoscalar screening masses is smaller than for the light quarks in the studied temperature region. At much higher temperatures, $T \gg m_{b}$, we expect that this difference will eventually approach the value of $0.3 T$ even for the bottomonium. Therefore, the increase in the difference between the vector and pseudoscalar screening masses shown in Fig. 2 is in fact expected and is consistent with an unbound bottom quark-antiquark pair. This again confirms our assertion that ground state bottomonium melts at $T>500 \mathrm{MeV}$. 


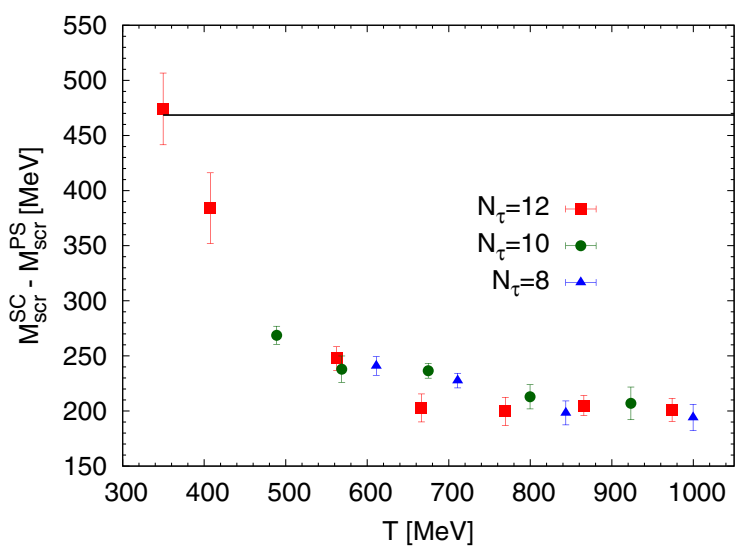

FIG. 3. The difference between the scalar and pseudoscalar screening masses as a function of the temperature obtained on lattices with $N_{\tau}=8,10$ and 12 . The solid line corresponds to the difference between the $\eta_{b}(1 S)$ and the $\chi_{b 0}(1 P)$ mass from PDG.

We now examine the difference between the $\eta_{b}$ and $\chi_{b 0}$, i.e., pseudoscalar and scalar screening masses, as well as the difference between the $\Upsilon$ and $h_{b}$ masses. Again we do not expect these observables to be sensitive to the small error in our determination of the bottom-quark mass. In Fig. 3 we show the difference between the scalar and pseudoscalar screening masses; the error on each data point is determined by adding the statistical and scale-setting errors in quadrature. As one can see from Fig. 3 the cutoff dependence of the result is mild compared to the estimated errors. The difference between the axial-vector and vector screening masses is very similar to the one shown in the figure, hence not shown explicitly. At the lowest temperature $T=350 \mathrm{MeV}$, the difference between the scalar and pseudoscalar (or between the axial-vector and vector) screening masses agrees with the differences between the $\chi_{b 0}(1 P)$ and $\eta_{b}(1 S)\left[h_{b}(1 P)\right.$ and $\left.\Upsilon(1 S)\right]$ masses reported in the PDG [41]. This again suggests that $\chi_{b 0}(1 P)$ and $h_{b}(1 P)$ states exist in the deconfined medium at $T \leq 350 \mathrm{MeV}$ with relatively small medium modifications. This is also consistent with the recent lattice results using NRQCD which show almost no medium mass shift of the $h_{b}(1 P)$ and $\chi_{b 0}(1 P)$ states $[29,30]$. For $T>350 \mathrm{MeV}$ the difference between the scalar and pseudoscalar (axialvector and vector) screening masses decreases with increasing temperature, initially very rapidly, by about a factor of 2 in the temperature region $350 \mathrm{MeV} \leq T \leq 600 \mathrm{MeV}$, and then more slowly for $T>600 \mathrm{MeV}$. At very high temperatures the splitting between the scalar and the pseudoscalar screening masses is expected to be extremely small. In the light quark sector, this observation is related to the restoration of chiral and the effective restoration of axial U(1) symmetry. For bottom quarks these symmetries are explicitly broken by the large value of the quark mass. However, for $T \gg m_{b}$ we expect that the difference between the scalar and pseudoscalar screening correlators will eventually vanish. Therefore, the first rapid drop in the difference between the scalar and the pseudoscalar screening mass shown in Fig. 3 is related to the melting of $\chi_{b 0}(1 P)$ and $h_{b}(1 P)$ states, while the subsequent slower decrease for $T>600 \mathrm{MeV}$ is related to the tiny effects of the bottom-quark mass and their eventual disappearance in the limit of very high temperatures.

\section{CONCLUSIONS}

We performed a first comprehensive study about the temperature dependence of the pseudoscalar, vector, scalar and axial-vector bottomonium screening masses on the lattice using a relativistic (HISQ) action for the bottom quarks. We scanned a wide range of the temperature ranging from 350 to $1000 \mathrm{MeV}$, and for most temperatures performed these calculations at three different lattice cutoffs corresponding to temporal extent $N_{\tau}=8,10$ and 12 of the lattices. We have found the lattice spacing dependence of our results is small compared to other sources of errors and thus does not effect our main conclusion. At the lowest temperature, all four screening masses agree with the corresponding bottomonium masses at zero temperature. For the axial-vector and scalar screening masses we find a rapid change as a function of temperature for $T>350 \mathrm{MeV}$, while for vector and pseudoscalar screening masses the corresponding thermal modifications occurs at a higher temperature, $T>450 \mathrm{MeV}$. The small thermal modifications of the ground state bottomonium screening masses $\left[\eta_{b}(1 S)\right.$ and $\Upsilon(1 S)]$ for $T<450 \mathrm{MeV}$ are consistent with the lattice calculations within the NRQCD formalism [25,26,28]. On the other hand we predict that the $1 P$ bottomonia will melt at temperatures, somewhere about $350 \mathrm{MeV}$, while the ground state bottomonia will melt at $T>500 \mathrm{MeV}$.

\section{ACKNOWLEDGMENTS}

P. P. was supported by U.S. Department of Energy under Contract No. DE-SC0012704. The work of J. H. W. was supported by the U.S. Department of Energy, Office of Science, Office of Nuclear Physics and Office of Advanced Scientific Computing Research within the framework of Scientific Discovery through Advance Computing (SciDAC) award Computing the Properties of Matter with Leadership Computing Resources, and by the Deutsche Forschungsgemeinschaft (DFG, German Research Foundation)-Projektnummer 417533893/ GRK2575 “Rethinking Quantum Field Theory.” S. S. gratefully acknowledges financial support from the Department of Science and Technology, Government of India, through a Ramanujan Fellowship. The numerical calculations have been performed on UQSCD clusters in JLab and FNAL. 
[1] T. Matsui and H. Satz, Phys. Lett. 178B, 416 (1986).

[2] R. Sharma, Eur. Phys. J. Special Topics 230, 697 (2021).

[3] X. Yao, Int. J. Mod. Phys. A 36, 2130010 (2021).

[4] A. Rothkopf, Phys. Rep. 858, 1 (2020).

[5] G. Aarts et al., Eur. Phys. J. A 53, 93 (2017).

[6] A. Mocsy, P. Petreczky, and M. Strickland, Int. J. Mod. Phys. A 28, 1340012 (2013).

[7] A. Bazavov, P. Petreczky, and A. Velytsky, Quarkonium at finite temperature, in Quark-Gluon Plasma 4, edited by R. C. Hwa and X.-N. Wang (World Scientific, Singapore, New York, Berlin, 2010).

[8] Y. Nakahara, M. Asakawa, and T. Hatsuda, Phys. Rev. D 60 , 091503 (1999).

[9] M. Asakawa, T. Hatsuda, and Y. Nakahara, Prog. Part. Nucl. Phys. 46, 459 (2001).

[10] M. Asakawa and T. Hatsuda, Phys. Rev. Lett. 92, 012001 (2004).

[11] I. Wetzorke, F. Karsch, E. Laermann, P. Petreczky, and S. Stickan, Nucl. Phys. B, Proc. Suppl. 106, 510 (2002).

[12] F. Karsch, S. Datta, E. Laermann, P. Petreczky, S. Stickan, and I. Wetzorke, Nucl. Phys. A715, 701 (2003).

[13] T. Umeda, K. Nomura, and H. Matsufuru, Eur. Phys. J. C 39, 9 (2005).

[14] S. Datta, F. Karsch, P. Petreczky, and I. Wetzorke, Phys. Rev. D 69, 094507 (2004).

[15] A. Jakovac, P. Petreczky, K. Petrov, and A. Velytsky, Phys. Rev. D 75, 014506 (2007).

[16] A. Mocsy and P. Petreczky, Phys. Rev. D 77, 014501 (2008).

[17] P. Petreczky, Eur. Phys. J. C 62, 85 (2009).

[18] W. E. Caswell and G. P. Lepage, Phys. Lett. 167B, 437 (1986).

[19] G. P. Lepage, L. Magnea, C. Nakhleh, U. Magnea, and K. Hornbostel, Phys. Rev. D 46, 4052 (1992).

[20] C. T. H. Davies, K. Hornbostel, A. Langnau, G. P. Lepage, A. Lidsey, J. Shigemitsu, and J. H. Sloan, Phys. Rev. D 50, 6963 (1994).

[21] S. Meinel, Phys. Rev. D 79, 094501 (2009).

[22] S. Meinel, Phys. Rev. D 82, 114502 (2010).

[23] T. C. Hammant, A. G. Hart, G. M. von Hippel, R. R. Horgan, and C. J. Monahan, Phys. Rev. Lett. 107, 112002 (2011); 115, 039901(E) (2015).

[24] R. J. Dowdall et al. (HPQCD Collaboration), Phys. Rev. D 85, 054509 (2012).

[25] G. Aarts, S. Kim, M. P. Lombardo, M. B. Oktay, S. M. Ryan, D. K. Sinclair, and J. I. Skullerud, Phys. Rev. Lett. 106, 061602 (2011).
[26] G. Aarts, C. Allton, T. Harris, S. Kim, M. P. Lombardo, S. M. Ryan, and J.-I. Skullerud, J. High Energy Phys. 07 (2014) 097.

[27] S. Kim, P. Petreczky, and A. Rothkopf, Phys. Rev. D 91, 054511 (2015).

[28] S. Kim, P. Petreczky, and A. Rothkopf, J. High Energy Phys. 11 (2018) 088.

[29] R. Larsen, S. Meinel, S. Mukherjee, and P. Petreczky, Phys. Rev. D 100, 074506 (2019).

[30] R. Larsen, S. Meinel, S. Mukherjee, and P. Petreczky, Phys. Lett. B 800, 135119 (2020).

[31] F. Karsch, E. Laermann, S. Mukherjee, and P. Petreczky, Phys. Rev. D 85, 114501 (2012).

[32] A. Bazavov, F. Karsch, Y. Maezawa, S. Mukherjee, and P. Petreczky, Phys. Rev. D 91, 054503 (2015).

[33] A. Bazavov and J. H. Weber, Prog. Part. Nucl. Phys. 116, 103823 (2021).

[34] E. Follana, Q. Mason, C. Davies, K. Hornbostel, G. P. Lepage, J. Shigemitsu, H. Trottier, and K. Wong (HPQCD and UKQCD Collaborations), Phys. Rev. D 75, 054502 (2007).

[35] A. Bazavov et al. (HotQCD Collaboration), Phys. Rev. D 90, 094503 (2014).

[36] A. Bazavov, H. T. Ding, P. Hegde, F. Karsch, C. Miao, S. Mukherjee, P. Petreczky, C. Schmidt, and A. Velytsky, Phys. Rev. D 88, 094021 (2013).

[37] H. T. Ding, S. Mukherjee, H. Ohno, P. Petreczky, and H. P. Schadler, Phys. Rev. D 92, 074043 (2015).

[38] A. Bazavov et al. (HotQCD Collaboration), Phys. Lett. B 795, 15 (2019).

[39] A. Bazavov, P. Petreczky, and J. H. Weber, Phys. Rev. D 97, 014510 (2018)

[40] A. Bazavov et al. (MILC Collaboration), Proc. Sci. LATTICE2010 (2010) 074 [arXiv:1012.0868].

[41] P. A. Zyla et al. (Particle Data Group), Prog. Theor. Exp. Phys. 2020, 083C01 (2020).

[42] P. Petreczky and J. H. Weber, Phys. Rev. D 100, 034519 (2019).

[43] M. Laine and M. Vepsalainen, J. High Energy Phys. 02 (2004) 004.

[44] P. Petreczky, C. Miao, and A. Mocsy, Nucl. Phys. A855, 125 (2011).

[45] Y. Burnier, O. Kaczmarek, and A. Rothkopf, J. High Energy Phys. 12 (2015) 101.

[46] V. Koch, E. V. Shuryak, G. E. Brown, and A. D. Jackson, Phys. Rev. D 46, 3169 (1992); 47, 2157(E) (1993).

[47] E. V. Shuryak, Rev. Mod. Phys. 65, 1 (1993).

[48] A. Bazavov et al., Phys. Rev. D 100, 094510 (2019). 